\title{
Early run II performance of the CMS Tracker
}

\author{
Brieuc Francois* ${ }^{* \dagger}$ \\ Université catholique de Louvain \\ E-mail: brieuc.francoisecern.ch
}

The CMS tracker is an all-silicon detector operating in the high-radiation environment presented by particle collisions in the LHC. This document discusses the early run II performance of both subcomponents, the Pixel and the Strip detectors. After a general presentation of the tracker, we detail the main modifications that were performed during the LHC first long shutdown and the commissioning operations that took place at the beginning of 2015. The status of the detector at the beginning of data taking is also reviewed and first results are presented to assess the readiness of this detector for run II.

The European Physical Society Conference on High Energy Physics

22-29 July 2015

Vienna, Austria

* Speaker.

${ }^{\dagger}$ On behalf of the CMS Collaboration. 


\section{Introduction}

The CMS detector [1] is a multi-purpose experiment taking place at the LHC complex. It has a nested structure with several sub-components arranged as follows. Going inward one can find the muon chambers, the superconducting magnet, the hadronic calorimeter, the electromagnetic calorimeter and finally the Silicon tracker, which is the central topic of these proceedings.

The CMS tracker is an all-silicon detector with an analogue readout and a sensitive area of about $200 \mathrm{~m}^{2}$ covering a pseudo-rapidity up to $2.5^{1}$. The purpose of this detector is to precisely measure the trajectories of the charged particles allowing also for an accurate reconstruction of primary and secondary vertices. The tracker is made of two independent components, the Strip detector and the Pixel detector. The latter is made of three barrel layers (BPix) distant of 4.4, 7.3 and $10.2 \mathrm{~cm}$ from the beam pipe and of four forward disks (FPix) at coordinates \pm 34.5 and \pm $46.5 \mathrm{~cm}$ on the $z$-axis. The BPix together with FPix are made of 1440 modules sharing 66 millions of pixels with a size of $100 \mu \mathrm{m} \times 150 \mu \mathrm{m}$. The Strip detector, surrounding the Pixel detector, is made of four inner barrel layers (TIB), six inner disks (TID), six outer barrel layers (TOB) and nine endcap disks (TEC) at each side of the TOB. This detector is made of 15148 modules with 9.6 millions $\mathrm{p}^{+}$-strips inserted on n-bulks. The modules were designed with a pitch between 80 and $205 \mu \mathrm{m}$ depending on the distance to the interaction point ensuring that the charges produced by crossing particles are collected on several strips, increasing thus the resolution. A detailed description of the CMS tracker can be found elsewhere [2].

\section{The LHC first long shutdown}

Both these detectors performed very well during run I but run II came with new challenges such as the increase in instantaneous and integrated luminosity inducing a more hostile radiation environment. In order to face this new challenge, the opportunity of the first long shutdown of the LHC (LS1) was taken to perform some ameliorations ${ }^{2}$.

The operating temperature of the Strip cooling plant during run I was $+4^{\circ} \mathrm{C}$ due to dew point limitations. Whereas this temperature was safe until LS1, the run II luminosity requires a lower operating temperature to mitigate the radiation damages. In order to allow operating colder the following elements were improved.

- A new dry-gas plant has been installed to augment the amount of dry gas supplied to the Tracker volume. This installation provides eight times as much dry gas as the previous system did, and will also allow fine-grained regulation of the flow.

- Several hundred high-precision sensors were installed to better monitor the humidity and temperature conditions during cold operation. In addition, all cooling bundles outside the

\footnotetext{
${ }^{1}$ The CMS experiment uses a right-handed coordinate system with the origin at the nominal collision point, the $x$-axis pointing to the centre of the LHC ring, the $y$-axis pointing up (perpendicular to the LHC plane), and the $z$-axis along the anticlockwise beam direction. The polar angle $(\theta)$ is measured from the positive $z$-axis and the azimuthal angle $(\varphi)$ is measured from the positive $x$-axis in the $x-y$ plane. The radius $(r)$ denotes the distance from the $\mathrm{z}$-axis and the pseudorapidity $(\eta)$ is defined as $\eta=-\ln \left[\tan \left(\frac{\theta}{2}\right)\right]$.

2 The run I refers to the data taking period from 2010 to 2012. It is immediately followed by the first long shutdown (2013-2014) and the run II started in 2015.
} 
Tracker itself were equipped with heater wires and temperature sensors to guarantee safe operation above the cavern dew point in the future.

- New methods for vapour-sealing and insulation were engineered to suppress humidity inside the Tracker volume.

A study based on simulation was conducted in order to choose the operating temperature for run II. The full depletion voltage after an integrated luminosity of $500 \mathrm{fb}^{-1}$ was compared in two scenarios with different operating temperature. In the first scenario we operate at $-15^{\circ} \mathrm{C}$ up to LS2 and at $-20^{\circ} \mathrm{C}$ afterwards, in the second scenario we operate at $-20^{\circ} \mathrm{C}$ from the beginning of run II onwards. The full depletion voltage difference between the two scenarios has been found to be, for all the modules, below $15 \mathrm{~V}$, which is small in comparison to the $\sim 400 \mathrm{~V}$ necessary at the end of the Strip detector lifetime. As a result, even if a colder temperature is achievable without any problem, the safe and less mechanically demanding choice of $-15^{\circ} \mathrm{C}$ was taken.

The other main project undertaken during LS1 is the repair of broken channels in the Pixel detector. At the end of run I, BPix and FPix were respectively affected by $2.3 \%$ and $7.8 \%$ of faulty channels. The Pixel detector has been designed to allow an easy extraction from CMS especially to make this kind of intervention possible. The extraction started in summer 2013, most of the faulty modules were replaced and the detector was reinserted in December 2014. At the beginning of run II, the active fractions were $99.98 \%$ for FPix and $98.01 \%$ for BPix, which is affected by a power supply problem appearing when switching on the magnet. The location of the BPix dead cells is such that at least two layers are active in any of the polar-azimuthal directions and thus they have a negligible impact on the track efficiency.

\section{Commissioning of the detector}

After the long period of shutdown during which important modifications took place, the detector had to be fully recommissioned in order to be ready for the beginning of run II. To begin with, the calibration of the lasers sending data across optical fibres was retuned. These lasers come with four different levels of signal amplification and are tuned in order to have a signal response in the front-end chip which is as homogeneous as possible. The signal gain in the read-out chain mostly depends on radiation damages and operating temperature. Since the Strip is now operating at $-15^{\circ} \mathrm{C}$, the run I settings were not optimal any more and had to be updated. Fig. 1 compares run I $\left(+4^{\circ} \mathrm{C}\right)$ laser settings on the left and run II $\left(-15^{\circ} \mathrm{C}\right)$ settings on the right. Two observations can be drawn from these plots. Firstly, as expected, we need a lower signal amplification when operating at lower temperature and secondly, we still have ample room to account for future radiation damages as very few modules operate at the highest possible gain at this stage.

This first step does not require specific data taking and thus could be realized in the early phase of commissioning. The second step began with the cosmic muon data taking period that took place at the beginning of 2015. These data were used to perform a first alignment, a coarse timing scan and to mask the noisy pixels. Since the $20^{\text {th }}$ of May, the LHC delivered proton proton collisions at a center of mass energy of $13 \mathrm{TeV}$ with a bunch crossing separation of $50 \mathrm{~ns}$. At the beginning of this new data taking period, several commissioning studies were performed, including a Pixel high voltage scan and a hit efficiency measurement, shown in Figure 2. The full hit efficiency is around 

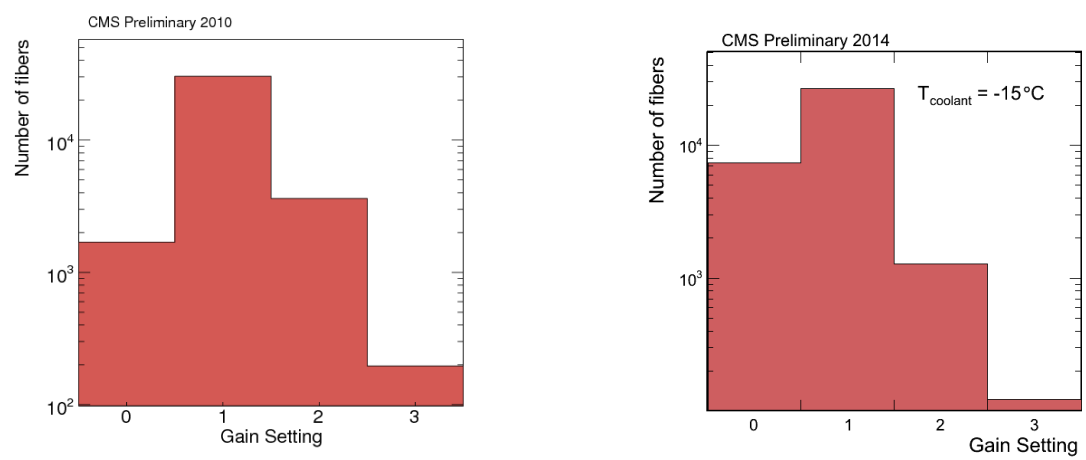

Figure 1: Number of optical fibres with respect to the laser gain setting. The picture on the left shows the setting used during run I when operating at a temperature of $+4^{\circ} \mathrm{C}$, the picture on the right shows the run II setting with an operating temperature of $-15^{\circ} \mathrm{C}$.

99.8\% and is reached at a voltage between 100 and $150 \mathrm{~V}$, depending on the layers and disks. The chosen operating voltage for FPix is $300 \mathrm{~V}$ as it was during run I. For BPix, the operating voltage has been raised from $150 \mathrm{~V}$ to $200 \mathrm{~V}$ to mitigate charge trapping effects while maintaining reasonable charge sharing.
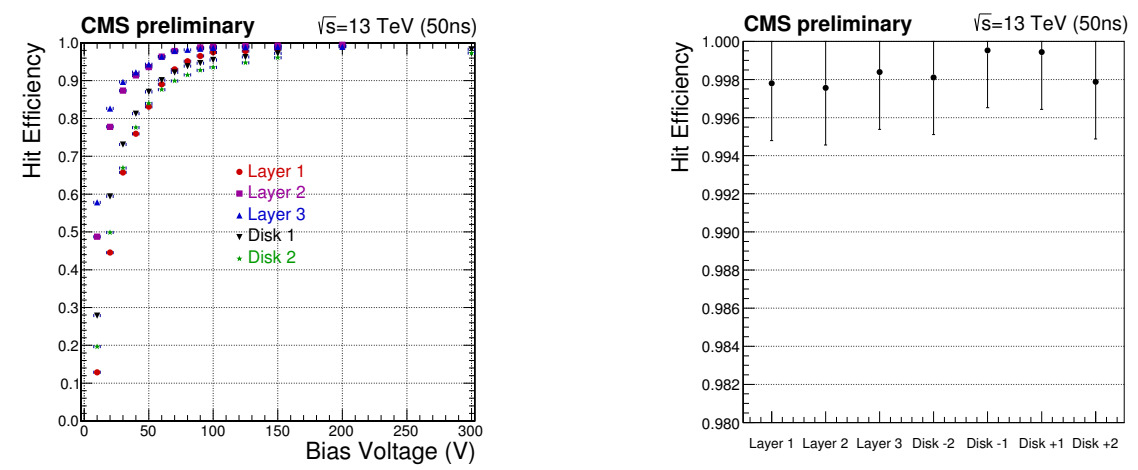

Figure 2: Example of commissioning results from the beginning of $13 \mathrm{TeV}$ proton proton collisions for the Pixel detector. The hit efficiency as a function of the voltage applied is shown on the left whereas the total hit efficiency at nominal voltage is shown on the right.

\section{Alignment}

The alignment of the Tracker consists of the simultaneous determination of about two hundred thousand parameters from a large sample of reconstructed charged particle trajectories. The central principle is the minimisation of the residuals defined as the difference between the reconstructed hit position and the predicted hit position from the track. This exercise was performed with several data samples taken in different conditions in order to minimize the geometric displacements for which the alignment procedure is blind (weak modes). In Figure 3 (top), one can see the $3 \mathrm{~mm}$ 
displacement of the BPix that was applied during its reinsertion in CMS in order to better center it with respect to the beam interaction point.
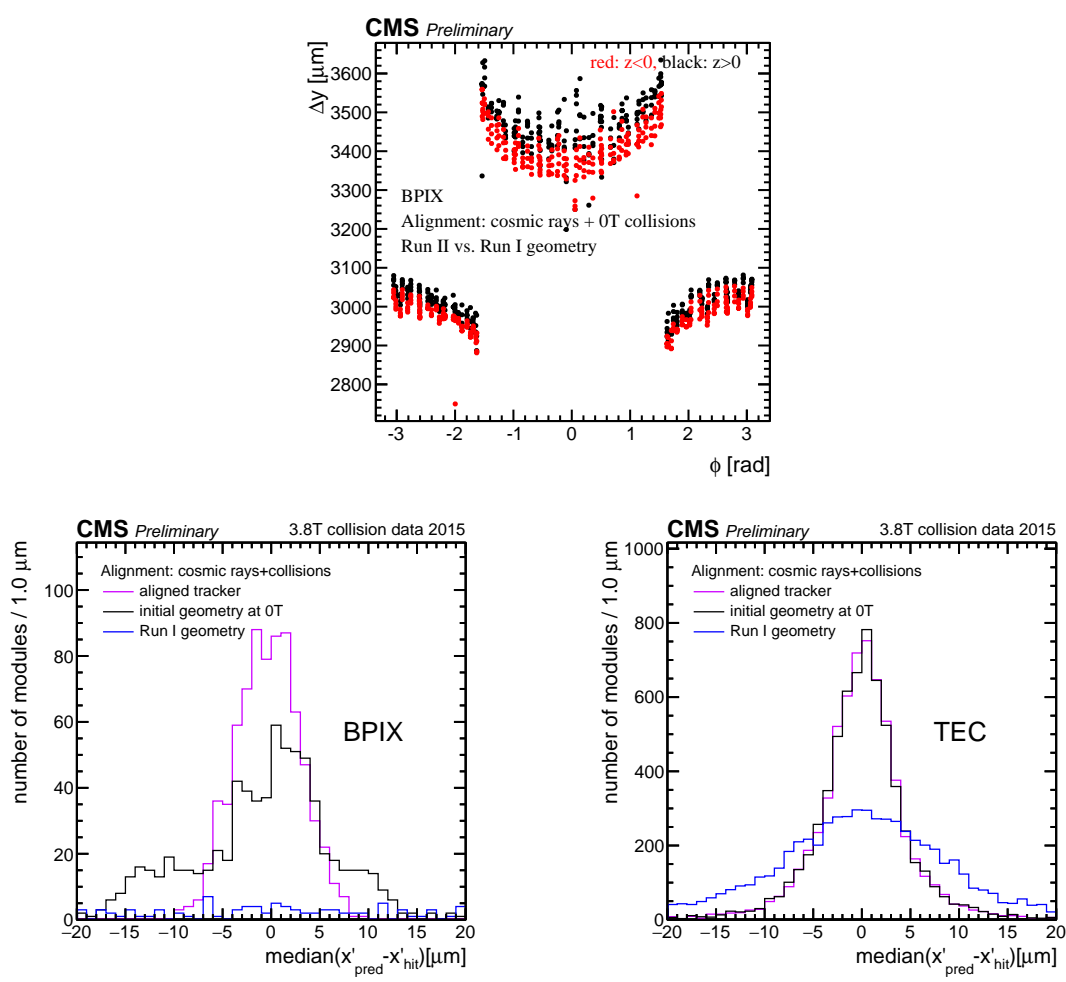

Figure 3: Top: difference between run I and run II geometry for the BPix after performing the alignment with the cosmic run data sample as well as the first proton proton collisions taken without magnetic field. The plot shows the coordinate difference in the direction perpendicular to the LHC plane as a function of the azimuthal angle of the considered module. Bottom: number of modules as a function of the DMR in a local module dependent direction in BPix (left) and TEC (right) for different sets of alignment parameters. The nominal alignment parameters obtained using both cosmic runs and proton collisions with or without magnetic field (purple curve) is compared to two other set of alignment parameters. In black, the one used to start the data taking with magnetic field (obtained from the collision runs without magnetic field) and in blue, the one from the end of run I.

Figure 3 (bottom) shows a subset of results from one of the three methods used to validate the alignment parameters [3]. The method considered here uses the distributions of medians of unbiased track-hit residuals (DMR) for each module. To extract the unbiased track-hit residual, the hit in question is removed from the track which is then re-fitted with the alignment parameters under consideration. Any deviation from zero on the DMR indicates a possible bias. The BPix DMR shows a clear improvement when going from run I or $0 \mathrm{~T}$ geometry to the new alignment. The important improvement with respect to run I geometry is due to the extraction of the Pixel detector and the amelioration with respect to $0 \mathrm{~T}$ geometry is expected as this sub-detector moves when the magnet is switched on. One can also see a clear improvement between run I and the current geometry in the TEC DMR, but this is mainly due to the interplay with the misalignment of the Pixel impacting the fitted tracks. 


\section{Detector readiness for run II}

In order to assess the readiness of the Tracker at the beginning of run II, one can consider a quantity derived using tracker only informations: the energy loss $\left(\frac{d E}{d X}\right)$ of the charged particles with respect to their momentum (Figure 4). To extract this quantity, one needs a proper alignment to derive the track momentum as well as its path through the sensors and a proper gain calibration to translate the output signal into an energy deposit. Looking at the positive $x$ axis one can distinguish several bands corresponding to the deuteron, proton and kaon going from right to left.

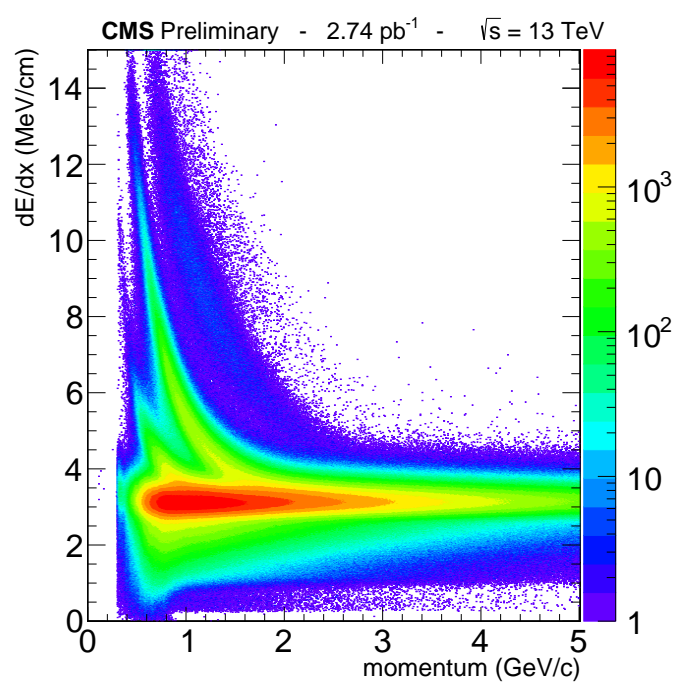

Figure 4: Charged particles' energy loss per unit of travelled length $\left(\frac{d E}{d X}\right)$ with respect to their momentum.

\section{Conclusion}

A tremendous work has been done during the first long shutdown of the LHC. To face the run II high luminosity challenge, the detector had to be operated at a colder temperature then during run I. To make this possible the cooling plants, the dry gas and the sealing system were significantly improved. Most of the defective Pixel components were replaced. The active fraction went from $92.2 \%$ to $99.98 \%$ in FPix and from $97.7 \%$ to $98.01 \%$ in BPix. The smaller relative improvement in active fraction of the BPix is mainly due to a power supply problem when switching the CMS magnet on. The detector was commissioned and aligned successfully during 2015 and was ready for the start of data taking at $13 \mathrm{TeV}$.

\section{References}

[1] The CMS Collaboration, The CMS experiment at the CERN LHC, 2008 JINST 3 S08004.

[2] The CMS Collaboration, Tracker Technical Design Report, CERN/LHCC 98-6 CMS TDR 5. Addendum to the CMS Tracker TDR, CERN/LHCC 2000-016.

[3] The CMS Collaboration, Alignment of the CMS Tracking-Detector with First 2015 Cosmic-Ray and Collision Data, CERN-CMS-DP-2015-029 